\title{
Un opérateur à peine franc-comtois
}

\author{
Várkonyi, Zsófia \\ Université d'Orléans, LLL \\ zazivac@gmail.com
}

\section{Observation introductive : un usage non-standard}

Lorsqu'on parle des particularités dialectales d'une langue, on pense le plus souvent à un ensemble formé d'un vocabulaire particulier (mots archaïques et/ou en patois), d'expressions caractéristiques, et parfois d'usages non-standards ou archaïques de la grammaire de la langue en question. Il est moins fréquent - et moins aisé - de relever les particularités qui consistent à utiliser les unités linguistiques de la langue standard mais en leur associant des significations différentes. Mettre en évidence et décrire ces divergences sémantiques devrait être, en principe, le travail des sémanticiens, mais les sémanticiens s'intéressent relativement rarement aux usages régionaux, les considérant comme des objets d'étude privilégiés de la sociolinguistique. Or, la sociolinguistique est justement à même de fournir un corpus potentiellement intéressant à la sémantique, qui peut de son côté produire des descriptions sémantiques adéquates aux éléments de ce corpus.

La présente étude sémantique porte sur une particularité dans la manière d'utiliser le morphème 'à peine' en Franche-Comté. Elle est appelée à être complétée par une analyse sociolinguistique : mon but n'est ni de marquer les limites géographiques de cet emploi, ni d'analyser les différences d'usage entre milieux urbain et rural, ou encore entre gens cultivés et gens peu instruits. J'ai tâché de me limiter aux aspects sémantiques du phénomène : l'observer et tenter d'en rendre compte au moyen d'un outil de description de la signification. Les tests que $\mathrm{j}$ 'ai faits auprès des locuteurs ne servent qu'à confirmer l'existence du phénomène observé et n'ont en aucun cas de valeur de statistique. J'exposerai néanmoins par la suite les résultats de ces tests qui permettent de concevoir dans quelles sortes de cas cet emploi particulier peut être attesté, et qui, j'espère, pourront intéresser des sociolinguistes et les inciter à en dresser une carte plus exacte $^{1}$.

Ces précautions étant prises, je me borne à signaler quelques occurrences d'énoncés attestées in situ ${ }^{2}$, occurrences pour le moins surprenantes :

(1) Je suis désolée, je suis à peine en retard.

(2) Je vous fais les croissants à moitié prix parce qu'ils sont à peine cuits. (Tout en montrant du doigt des croissants légèrement noircis.)

(3) Je dois me trouver une flûte en La : celle en Sol est à peine grave.

On remarquera que dans les énoncés cités ci-dessus, il ne s'agit pas seulement de l'utilisation de l'expression 'à peine' dans un sens légèrement inhabituel, on a l'impression que le choix de l'expression est complètement inadapté. L'énoncé (1) est difficile à interpréter en français standard, le (2), quand on connaît la situation d'énonciation, est quasiment impossible ; et pour peu que l'on ait quelques notions musicales et que l'on sache que la gamme de Sol est plus grave que celle de La, le (3) l'est également. Étant donné que ces énoncés n'avaient pas l'air de provoquer de problèmes de compréhension au moment de leur production, l'observateur est amené à admettre que l'expression doit avoir une signification différente en français dialectal, plutôt que de penser que les Francs-Comtois utilisent mal le français.

« Significations différentes » ne veut pas nécessairement dire qu'il n'existe aucun rapport entre celle en français standard et celle en franc-comtois. Mon but ici est de mettre en évidence une polysémie qui, incontestablement, comprend une ascendance commune des deux versions étudiées. Néanmoins, l'étude de cette filiation relève davantage de l'étymologie que d'une analyse sémantique synchronique. La description sémantique que je propose pour l'expression dialectale en termes de contraintes argumentatives - description dont les avantages seront détaillés plus loin - n'exclut pas pour autant la 
possibilité d'un niveau plus général de description qui pourrait rendre compte de tous les emplois, selon la démarche préconisée par (Némo \& Berruecos, 2003).

Avant de procéder à l'étude du morphème - en commençant par celui en français standard, afin de le contraster avec celui en français dialectal - il convient d'expliciter quelle est la notion de sémantique dont je me sers ici. Il est en effet indispensable de préciser pourquoi cette étude des emplois de 'à peine' relève non pas de la pragmatique mais bien de la sémantique.

\subsection{La construction du sens}

Nous avons vu que l'usage franc-comtois de 'à peine' pose un problème d'interprétation, donc de construction de sens pour les locuteurs de français standard. L'interprétation d'un énoncé est conditionnée par des facteurs linguistiques et extralinguistiques. Comme cet article se réclame de la sémantique, il est nécessaire avant tout de déterminer lesquelles d'entre eux sont les facteurs pertinents pour une analyse sémantique. Le schéma dressé par (Raccah, 2011) rend compte des différentes sortes d'apports à la construction du sens :

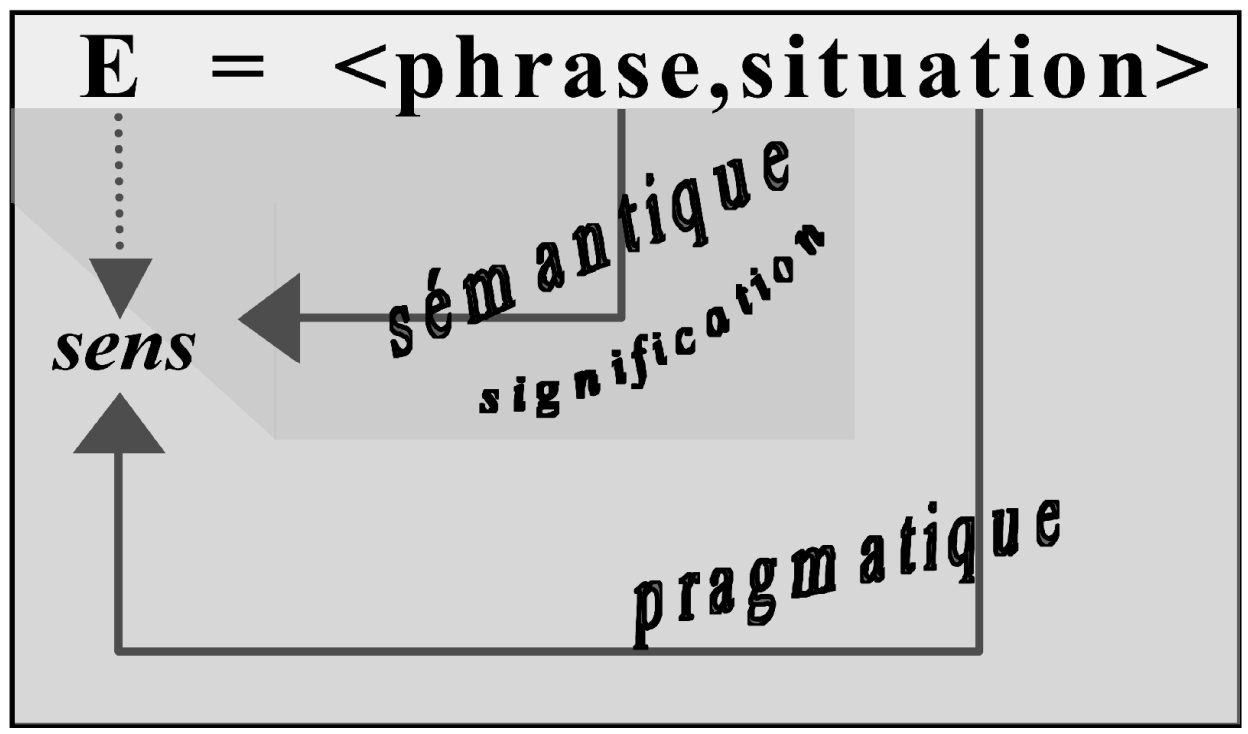

Figure 1 : Selon ce schéma, l'énoncé E est complètement caractérisé par le couple <phrase, situation>. Le terme phrase correspond à l'unité linguistique abstraite dont l'énoncé est une actualisation. La situation englobe tous les éléments, variables ou stables, qui constituent les circonstances de l'énonciation. Le sens est constitué à la fois de l'effet de ces éléments et l'effet de ce qui vient de la phrase même. Le terme signification est réservé à ce qui, dans les unités linguistiques abstraites, contraint la construction du sens.

Notons que s'agissant d'un concept construit, et construit selon les besoins des chercheurs, la notion de sémantique n'est pas une entité unie et objective. Les différentes notions de sémantique s'appuient sur des modèles distincts de description de la langue, dont l'élaboration a toujours été conditionnée par des considérations théoriques et métathéoriques diverses. La définition même de la sémantique peut varier selon les divergences terminologiques. Mon but ici n'est pas de les examiner en détail, mais de choisir le type de sémantique qui s'avérera pertinent pour ce travail.

La sémantique, dans l'optique du schéma présenté ci-dessus, est « la science des contraintes que les unités linguistiques imposent sur la construction du sens des énoncés de ces unités. » (Raccah, $2005: 1$ ) Les contraintes d'autres types sont étudiées non pas par la sémantique mais par la pragmatique. 
Le schéma ci-dessus (Fig. 1) représente clairement le fait que si les éléments venus de la situation d'énonciation influencent la construction du sens, cela se passe sans qu'il y ait interférence avec les éléments venus de la langue. En effet, ils ne feront que s'ajouter à la signification de la phrase, sans modifier celle-ci. Le sens est construit de l'ensemble de ces éléments, mais à partir d'un noyau stable qui est la signification. Une vaste littérature, d'Austin à Ducrot, témoigne de résultats de recherches qui montrent que la signification ne peut être réduite à la seule dénotation. Même Fauconnier, qui a pourtant subi l'influence de la linguistique cognitive américaine, très marquée par une certaine forme de dénotationnalisme ${ }^{3}$, dans (Fauconnier, 1984 : 9) rejette la vision «ferroviaire » où les mots seraient des wagons et les phrases des trains qui doivent acheminer pour qu'on les reçoive et que l'on examine leur contenu. Ainsi, des auteurs comme Ducrot ou Culioli considèrent la signification plutôt comme un ensemble d'instructions que les unités linguistiques fournissent à la construction du sens. Selon la formulation de Ducrot :

\begin{abstract}
«Elle [i.e. la signification] contient surtout, selon nous, des instructions données à ceux qui devront interpréter un énoncé de la phrase, leur demandant de chercher dans la situation de discours tel ou tel type d'information et de l'utiliser de telle ou telle manière pour reconstruire le sens visé par le locuteur. » (Ducrot et alii, 1980b : 12)
\end{abstract}

Dans cette optique, une description sémantique doit obligatoirement comporter des indications concernant des instructions stables de l'unité linguistique décrite.

\title{
2 'À peine’ en français standard
}

Pour que l'observateur de l'emploi dialectal de 'à peine' soit en mesure de dire en quoi ledit emploi se différencie de l'usage standard, il doit disposer d'une description sémantique de ce morphème en français standard, description permettant de rendre compte de cette différence. J'entends par là qu'elle ne peut pas être réduite à la simple valeur logique de l'unité de langue en question, car la valeur logique n'explique pas pourquoi l'interlocuteur non-averti se trouve dans la difficulté, voire dans l'impossibilité d'interpréter des énoncés comme (1), (2) et (3). En effet, pour tout énoncé, les conditions de vérités de à peine $X$ sont le mêmes que celles de $X$, ce qui ne donne aucune indication sur la raison pour laquelle on trouve normal l'énoncé

(1') Je suis désolée, je suis en retard.

et bizarre l'énoncé

(1) Je suis désolée, je suis à peine en retard.

\subsection{Valeur informative vs valeur discursive}

Avant même ses premiers travaux sur l'argumentation dans la langue (AdL), Oswald Ducrot s'intéresse à l'expression 'à peine' qu'il traite habituellement en paire avec 'presque'. Dans (Ducrot, 1972), il remarque un premier fait qui indique que ces deux opérateurs requièrent une description différente de leurs valeurs logiques respectives :

$$
\begin{aligned}
& \text { «à peine et presque [...] nous semblent avoir ceci en commun que leur introduction } \\
& \text { dans un énoncé permet de présupposer un certain fait, et poser une appréciation sur } \\
& \text { l'importance de ce fait. »(Ducrot, } 1972: 254)
\end{aligned}
$$

Le test des enchaînements discursifs en donc et en pourtant lui permettent d'observer que lorsque l'on fait suivre un énoncé contenant un 'à peine $X$ ' où $X$ est l'entité sur laquelle porte 'à peine ${ }^{, 4}$, les enchaînements ne concernent pas directement X. Les suites possibles de l'énoncé Jacques était à peine en retard sont «par exemple Pourtant, le train était déjà parti, ou Jacques avait donc quelques chances d'avoir le train. » (Ducrot, $1972: 255)$

Le test des enchaînements reste sa méthode de travail privilégiée tout au long de ses travaux ultérieurs, notamment dans ceux sur les potentialités argumentatives de la langue. Ces potentialités sont, pour Ducrot et ses successeurs, bien plus qu'une part rhétorique qui s'ajoute en amont à la signification 
«purement linguistique »: il s'agit d'un aspect fondamental de la sémantique. Comme le souligne Raccah :

\begin{abstract}
«[...] même si tous les énoncés ne sont pas nécessairement des argumentations, il n'en reste pas moins que toute phrase peut être utilisée dans un énoncé qui est une argumentation. Une conséquence théorique essentielle de ce fait est qu'une description sémantique des phrases (et donc de leurs constituants) doit nécessairement rendre compte de leurs utilisations dans des énoncés qui constituent des argumentations : une théorie adéquate de la signification doit, au minimum, comporter un volet argumentationnel.» (Raccah, 2002)
\end{abstract}

Dans les discours, les enchaînements des énoncés suivent des règles internes qui sont basées, comme Ducrot le souligne, non pas sur l'état du monde mais sur le sens des énoncés. L'auteur explicite ainsi le rapport entre argumentation et suites discursives :

«[...] le thème central de la théorie argumentative est que le sens d'un énoncé contient une allusion à son éventuelle continuation : il lui est essentiel d'appeler tel ou tel type de suite, de prétendre orienter le discours ultérieur dans telle ou telle direction. S'il est argumentatif, ce n'est pas seulement par ce qu'il dit sur le monde, mais par ce qu'il est, si on le considère en lui-même. » (Ducrot, 1980a : 11)

La méthode de l'AdL pour déterminer les possibilités argumentatives des phrases consiste alors à les tester dans des enchaînements discursifs. La possibilité ou l'impossibilité (ou plutôt l'étrangeté) des enchaînements répond à la question de savoir si telle ou telle potentialité argumentative fait partie, ou non, de la signification de la phrase.

Dans ses travaux, Ducrot défend l'idée que

- la valeur argumentative des énoncés est indépendante de la valeur informative et

- la valeur argumentative est même primordiale, puisque l'analyse argumentative est susceptible de rendre compte des phénomènes que la logique est impuissante à décrire ${ }^{6}$.

Ainsi, les opérateurs 'à peine' et 'presque' seront souvent utilisés pour illustrer cette indépendance de la valeur informative, notamment dans (Ducrot, 1980) et (Anscombre \& Ducrot, 1983).

Les exemples cités dans ces travaux montrent que, bien que, en considérant l'information véhiculée, 'à peine $X$ ' a les mêmes conditions de vérité que $\mathrm{X}$, et 'presque $X$ ' a les mêmes conditions de vérité que non $\mathrm{X}$, leur comportement argumentatif est le contraire de ce que l'on pourrait attendre d'après les conditions de vérité : 'à peine $X$ ' et 'non $X$ ' peuvent servir la même conclusion non $r$ et similairement, 'presque $X$ ' et $X$ argumentent tous les deux dans le sens de $r$. La comparaison des deux énoncés suivants, contenant respectivement 'presque' et 'à peine', permet d'observer les comportements argumentatifs de ces opérateurs :

(4) * Il fait à peine nuit, allume tes veilleuses.

(5) * Il fait presque nuit, allume seulement tes veilleuses. ${ }^{7}$

Dans les deux cas, les suites sont logiquement correctes, mais contraires à l'esprit de la langue. L’inversion des deux enchaînements rendrait les deux énoncés énonçables.

\title{
2.2 Le modèle topique dans la description des articulateurs
}

Alors que les premiers modèles issus des travaux sur l'argumentation étaient opérationnels pour la description des connecteurs et les opérateurs, le modèle topique, que Ducrot avait conçu avec Jean-Claude Anscombre (Ducrot, 1988) et mis au point par la suite avec Pierre-Yves Raccah, a permis dans un premier temps la description des énoncés contenant des articulateurs déjà décrits, ensuite la description des éléments de lexique autres que les articulateurs. La version topique était ensuite développée par Raccah, conduisant à la Sémantique des Points de Vue (Raccah, 1996). Dans le cadre limité du présent article, je 
dois me borner à une brève évocation des seuls aspects de ces recherches qui sont pertinents pour les objectifs de cette étude ${ }^{8}$.

Selon le modèle topique, pour que les argumentations puissent être comprises, des principes d'inférence, propres à une communauté linguistique servent de garants. Ils assurent le passage entre les arguments et les conclusions que ceux-ci étayent. Les argumentations possibles sont délimitées par le choix des mots, qui, eux-mêmes, évoquent certains de ces principes. Ces thèses sont habituellement illustrées par l'exemple suivant, devenu classique :

(6) Il fait beau mais j'ai du travail.

Où la situation d'énonciation est une proposition de promenade à laquelle l'énoncé (6) est la réponse. Le premier segment de l'énoncé (Il fait beau) est, dans la situation donnée, un argument en faveur de la promenade, le deuxième segment ( $j^{\prime}$ 'ai du travail) est un argument contre la promenade. ${ }^{9}$ Les garants qui permettent de comprendre en faveur de quelles conclusions ces segments sont destinés correspondent aux principes :

g1) « le beau temps est favorable à la promenade »

g2) « le travail est défavorable à la promenade ».

g1 et $g 2$ sont des formulations possibles des principes qui sont généralement acceptés par une communauté linguistique. Comme en général ces principes ne sont pas explicités dans le discours, la manière précise dont on les formule ici n'a pas beaucoup d'importance. Tous ces principes, appelés topoi en référence à la notion aristotélicienne, ont trois caractéristiques en commun : ils sont

- présentés comme généraux (c'est-à-dire comme applicables à d'autres situations que celle de l'énonciation),

- présentés comme partagés (c'est-à-dire comme acceptés par une communauté dont l'interlocuteur fait également partie),

- graduels.

En ce qui concerne les deux premiers caractéristiques, les topoi ne sont pas nécessairement généraux et partagés, mais ils sont toujours présentés comme tels, sans quoi ils ne pourraient pas servir de garant. Le caractère graduel des topoi, en revanche, découle de leur nature. (Bruxelles, Ducrot \& Raccah, $1994: 88$ ) présentent l'exemple suivant pour illustrer cette thèse :

Les énoncés de la phrase «Il a certainement gelé cette nuit, car les plantes sont mortes » s'appuient sur le principe qui établit un lien entre le gel et la mort des plantes. Les énoncés de la phrase « Il a dû faire très froid cette nuit, car les plantes sont en mauvais état » invoquent une autre règle, reliant le froid avec le mauvais état des plantes. Ces deux règles portent sur des degrés distincts des mêmes propriétés (degré de froid, degré de mauvais état). Les topoi étant des généralisations de ce type de garants d'argumentation individuels, ils sont eux-mêmes graduels. Le topos généralisant ces deux garants (et d'autres, figurant sur la même échelle) serait

«Plus il fait froid, plus les plantes sont en mauvais état. »

La version topique de l'AdL défend l'hypothèse selon laquelle tous les topoi peuvent être réduits à la même forme. Les topoi sont formalisés de la façon suivante :

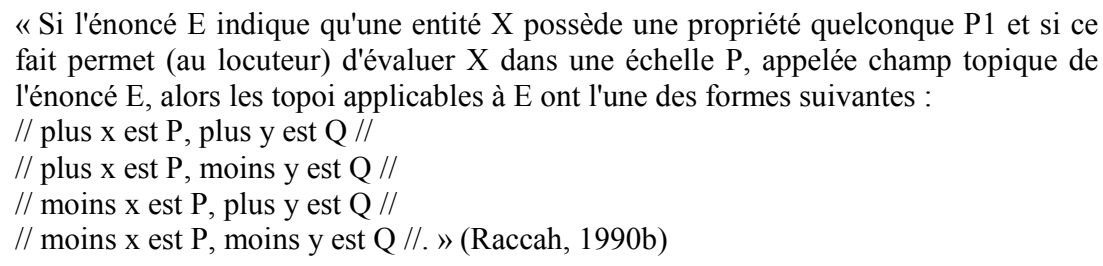

On utilise également la formulation courte 


$$
\begin{aligned}
& / /+\mathrm{P},+\mathrm{Q} / / \\
& / /-\mathrm{P},-\mathrm{Q} / / \\
& / /+\mathrm{P},-\mathrm{Q} / / \\
& / /-\mathrm{P},+\mathrm{Q} / /
\end{aligned}
$$

où $\mathrm{P}$ et $\mathrm{Q}$ sont des champs topiques, + et - sont les signes des champs topiques. +/-P est appelé l'antécédent du topos, +/-Q le conséquent du topos.

Le principal intérêt que présente la version topique de l'AdL est qu'elle a rendu possible la description sémantique des mots " ordinaires ». Il en a résulté que la description des articulateurs, si elle n'a pas été tout à fait reléguée au second plan, n'a pas été pour autant systématiquement mise à jour à la lumière des résultats théoriques plus récents. Des descriptions en termes de contraintes sur les topoi ont été néanmoins réalisées par Raccah pour plusieurs connecteurs et opérateurs.

Si l'on procède à une description de 'à peine' en ces mêmes termes, on remarquera que cet opérateur contraint le choix des formes de topoi de la même manière que l'opérateur 'peu'. En effet, dans les phrases comportant 'peu $X$ ', pour toute situation, le signe de l'antécédent du topos évoqué par X sera $(\text { moins })^{10}$, et ceci reste valable si l'on remplace 'peu $X$ ' par 'à peine $X$ '. A titre d'exemple, dans

\section{(7) Jean a peu bu.}

tout comme dans

(8) Jean a à peine bu.

le topos évoqué sera quelque chose comme // Moins on boit, plus on est capable de conduire //, ou encore // Moins on boit, moins on dit des bêtises //.

Le fait que les contraintes sont les mêmes n'implique pas pour autant que 'peu' et 'à peine' sont des synonymes. Comme Ducrot le remarque dans (Ducrot, 1972), certains emplois de 'à peine' ressemblent à un 'peu' et certains plutôt à un 'seulement'. Dans Julie a à peine seize ans, 'à peine' pourrait être remplacé par un 'seulement' mais non pas par un 'peu'. On remarquera au passage que 'seulement' - qui ne peut pas être considéré comme un synonyme de 'peu'- fait également partie des unités linguistiques qui exigent un antécédent de topos dans le champ moins. Les topoi évoqués dans

(9) Julie a à peine seize ans.

et dans

(10) Julie a seulement seize ans.

sont du type // Moins on est âgé, moins on a de l'expérience // ou // Moins on est âgé, plus on a de l'énergie //.

La description calquée sur celle de 'peu' est donc la suivante :

Dans les phrases comportant 'à peine $X$ ', pour toute situation, le signe de l'antécédent du topos évoqué par X sera - (moins).

Une fois équipé d'une description du morphème français (standard) 'à peine' qui donne les indications nécessaires concernant les suites discursives possibles et les contraintes sur les topoi utilisables, on est à même de la confronter à l'usage dialectal de ce morphème.

\section{3 'À peine' en Franche-Comté}

Les observations répétées des occurrences orales d'un emploi non-standard de 'à peine' en FrancheComté m'ont permis de dresser des hypothèses spontanées que j'ai dû consolider ensuite, d'une part par le biais d'un questionnaire réalisé auprès des locuteurs francs-comtois, d'autre part en construisant des 
tests 'en laboratoire'. Avant de présenter les hypothèses et leur consolidation, il convient de faire brièvement le point sur la différence de statut entre les méthodes de travail évoquées.

\subsection{Observation in situ, corpus constitué et 'travail de laboratoire'}

L'observation spontanée d'un fait linguistique fournit rarement un corpus cohérent, prêt à être analysé. Elle a comme principal rôle de mettre le chercheur sur la piste d'un objet d'étude potentiellement intéressant, elle permet de poser une première série de questions auxquelles il convient de chercher la réponse en priorité, comme par exemple : S'agit-il d'un vrai fait linguistique ou du produit d'un fait d'une autre nature qui n'intéresse pas la linguistique ? Existe-t-il un moyen d'étudier ce fait de manière scientifique et empirique? Quel est ce moyen?

Le corpus constitué, c'est-à-dire sélectionné selon des critères pertinents pour l'étude en question peut sembler la partie la plus objective du travail du linguiste. Or, malgré les avantages que présente l'observation contrôlée d'un ensemble constitué, c'est précisément l'illusion d'objectivité que le chercheur doit prendre avec toutes les précautions nécessaires. Le relevé de la récurrence d'un fait de langue permet d'étayer des hypothèses de manière inductive, mais ne peut en aucun cas justifier des relations de cause à effet.

Bien que les relations causales ne soient pas observables, des hypothèses abductives sur ces relations peuvent être testées par des manipulations sur des exemples construits. C'est ce que je ferai au paragraphe 4 , après avoir présenté les hypothèses et le questionnaire dans les lignes suivantes.

\subsection{Hypothèses}

La première hypothèse sur l'usage dialectal de 'à peine' concerne son existence. Les occurrences attestées pourraient être dues à un défaut de maîtrise de la langue française de la part de ses locuteurs ou à des accidents de langage, mais mon hypothèse consiste à supposer que :

(H1) Il existe un usage régional du morphème 'à peine' en Franche-Comté, qui exige une description sémantique différente de celle du morphème standard.

Comme j'ai souligné dans 3.1, la récurrence du fait observé, ainsi qu'un test réalisé en questionnant les locuteurs peuvent étayer ce type d'hypothèse de manière inductive, ce qui permet d'envisager la rationalité de l'hypothèse. En effet, nous verrons plus loin que les résultats du test que j'ai fait auprès des locuteurs confirment raisonnablement mon hypothèse.

La deuxième hypothèse part de l'observation selon laquelle dans les énoncés difficilement interprétables, l'opérateur 'à peine' pourrait être remplacé par 'un peu', opération conduisant à un énoncé qui cesserait d'être difficilement interprétable. Par exemple,

(1') Je suis désolée, je suis un peu en retard

est une phrase tout à fait normale.

(H2) L'usage dialectal ne concerne que les cas où, en français standard, 'à peine' pourrait être remplacé par 'peu', et dans ce cas il est utilisé dans le sens de 'un peu' ; les emplois de 'à peine' où il pourrait être remplacé par 'seulement' restent identiques à l'emploi standard.

À l'instar des remarques concernant $(\mathrm{H} 1)$, les réponses au questionnaire que j'ai réalisé confirment cette hypothèse. Dans les cas où 'à peine' ne pourrait pas être remplacé par 'peu' ou par 'un peu', la totalité des locuteurs questionnés utilisent 'à peine' de manière standard.

La vérification de la troisième hypothèse demande un 'travail de laboratoire'.

(H3) Si, en français standard, 'à peine' impose les mêmes contraintes sur l'utilisation des topoi que 'peu', la description de l'emploi dialectal de 'à peine' impose les mêmes contraintes que 'un peu'. 
J'examinerai la validité de cette hypothèse après avoir présenté le test réalisé auprès des locuteurs francscomtois et les résultats issus de ce test.

\subsection{Le questionnaire}

Le questionnaire (Voir Annexe) que j'ai élaboré pour confirmer ou infirmer la rationalité des hypothèses (H1) et (H2) se compose de trois types de questions :

1. des débuts de phrases comportant 'à peine', à continuer librement ;

2. des phrases comportant à la fois 'à peine' et un enchaînement, que les locuteurs doivent classer dans une des trois catégories : A) correct B) incorrect, le questionné ne pourrait jamais entendre ou dire ça $\mathrm{C}$ ) on pourrait entendre ça à l'endroit où le questionné vit mais il ne le dirait pas ;

3. une question directe sur la différence entre une phrase comportant 'à peine' et la même phrase où 'à peine' est remplacé par 'un peu'.

Les questionnés avaient la possibilité d'ajouter des remarques ou signaler si une question leur posait problème.

Le questionnaire à été soumis à quatre groupes de personnes :

- Groupe (a) : ceux qui sont nés en Franche-Comté et qui y vivent encore (26 personnes)

- Groupe (b) : ceux qui sont nés en Franche-Comté mais qui n’y vivent plus (6 personnes)

- Groupe (c) : ceux qui sont nés ailleurs mais qui vivent en Franche-Comté depuis de nombreuses années (13 personnes)

- Groupe (d) : ceux qui sont nés ailleurs et qui vivent ailleurs (10 personnes).

Étant donné qu'il ne s'agissait pas d'une étude à valeur statistique, je n'ai pas créé de catégorie à part pour ceux qui sont nés ailleurs, qui vivent ailleurs mais qui ont passé la plus grande partie de leur vie en Franche-Comté : je les ai comptés avec le groupe (c). D'ailleurs, si le groupe (a) a massivement manifesté l'emploi régional et le groupe (d) quasiment pas du tout, les deux groupes intermédiaires ont produit des résultats assez similaires, c'est-à-dire une quantité moins importante d'usage régional que le groupe (a) mais nettement plus importante que le groupe (d). Je n'ai pas fait de différence entre personnes habitant en ville ou en campagne, cultivés ou non. Néanmoins, comme c'était assez prévisible pour une étude portant sur un usage régional, on pouvait remarquer que les personnes habitant à la campagne et n'ayant pas eu beaucoup d'éducation (donc n'ayant pas été beaucoup confrontées à l'usage standard) produisent plus souvent l'emploi dialectal.

Sans exposer en détail la totalité des 20 questions et les réponses reçues, je me limite à en relever celles qui se sont avérées significatives pour la présente étude.

Le début de phrase Il fait à peine froid, donc... a été complété par $60 \%$ des sujets francs-comtois (groupe (a)) par une suite de type « il faut mettre un pull plus chaud», "je mets mon manteau » etc. 33\% du groupe (b), 25\% du groupe (c) et seulement 10\% du groupe (d) a répondu dans ce sens (c'est-à-dire une seule personne pour le groupe (d), ce qui peut être raisonnablement considéré comme un accident).

La phrase Je suis désolée, je suis à peine en retard a été jugé correcte par $34 \%$ des sujets du groupe (a), et la phrase Il y a à peine de vent, le linge va vite sécher par $27 \%$ des sujets du même groupe. Aucun sujet du groupe (d) n'a accepté ces deux phrases et seulement environ 10-15\% des deux groupes intermédiaires les ont jugées acceptables. Il est intéressant de remarquer que il y a à peine de vent, même amputé de son enchaînement, n'est pas correct en français (il faudrait plutôt dire le vent souffle à peine), mais la phrase ne semble pas être considérée comme incorrecte en franc-comtois.

La phrase Viens à peine plus près, s'il te plaît a été acceptée par $100 \%$ des sujets francs-comtois, par $100 \%$ des sujets qui sont nés en Franche-Comté mais qui n’y vivent plus (groupe (b)), par 70\% ceux qui 
ne sont pas d'origine franc-comtoise mais qui y vivent (groupe (c)), et seulement par 16\% de non FrancsComtois.

Le même genre de résultat était obtenu concernant la phrase Le café est à peine trop chaud, il faut que tu attendes avant de le boire : $100 \%$ du groupe (a), 83\% du groupe (b) et 100\% du groupe (c) l'ont trouvée acceptable, contre $16 \%$ du groupe (c). Une étude psycholinguistique pourrait mettre en évidence un éventuel biais d'interprétation dû à la présence de 'trop', qui pèserait plus dans le choix de la suite que la présence de 'à peine'. Vérifier cette hypothèse n'est ni mon but ni de mon ressort, mais il est utile d'envisager la possibilité de ce type de biais qui peut expliquer certains résultats inattendus.

La réponse $\mathrm{C}$ ) (on pourrait entendre ça à l'endroit où le questionné vit mais il ne le dirait pas) a été nettement plus largement donnée par les sujets des groupes (a), (b) et (c) que par ceux du groupe (d) quand il s'agissait de juger des emplois dialectaux.

Les réponses ci-dessus ne peuvent pas être considérées comme probantes - on ne peut jamais complètement exclure la possibilité que ces résultats soient dus à des causes différentes de celle proposée dans l'hypothèse $(\mathrm{H} 1)$ - tout ce que l'on peut dire c'est qu'elles sont compatibles avec cette hypothèse.

Comme prévu, aux questions comportant un 'à peine' qui ne peut pas être remplacé par 'peu' ni par 'un peu', les réponses étaient identiques pour les quatre groupes, aucun emploi régional n'a été relevé. Ainsi, la phrase Son travail à peine fini, Jean part pour son cours d'anglais a été jugé correcte par $100 \%$ des sujets des quatre groupes.

Le test a également révélé que, pour une raison qui reste encore à trouver, dans les constructions contenant 'à peine + Verbe', l'expression 'à peine' est utilisée de manière standard. Ce fut un résultat inattendu mais qui ne met pas en cause la rationalité des hypothèses testées. Ainsi, on peut conclure que l'hypothèse $(\mathrm{H} 2)$ n'a pas été infirmée par ces réponses.

\subsection{Le problème des jugements introspectifs}

Lorsque les sujets avaient à répondre explicitement à la question de savoir si, à leur avis, il y a une différence de sens entre Je suis à peine enrhumé et Je suis un peu enrhumé, et si oui en quoi elle consiste, les réponses des questionnés des quatre groupes ne correspondaient pas vraiment à ce à quoi on aurait pu s'attendre. Étant donné le pourcentage important des Francs-Comtois qui reconnaissaient et acceptaient l'usage dialectal de 'à peine' dans les autres parties du questionnaire, et la quasi-absence des non FrancsComtois qui acceptaient ce même usage, on pouvait s'attendre à ce qu'il y ait nettement plus de FrancsComtois que de non Francs-Comtois qui ne perçoivent pas de différence de sens entre les deux phrases. Or, la répartition des réponses selon les différents groupes semblait plutôt aléatoire. Il y avait des sujets dans les quatre groupes qui trouvaient qu'il n'y avait pas de différence : 34\% dans le groupe (a), 33\% dans le groupe (b), $40 \%$ dans le groupe (c) et $20 \%$ dans le groupe (d). Parmi ceux qui trouvaient qu'il y avait une différence, certains proposaient que c'était une différence de degré, d'autres que 'Je suis un peu enrhumé' était plus correct que 'Je suis à peine enrhumé', et certains (pas moins parmi les FrancsComtois que parmi les autres) avançaient la «bonne » réponse (pour le français standard), c'est-à-dire que la différence concernait les conséquences / l'appréciation / les conclusions possibles du fait d'être enrhumé. Les mêmes personnes qui utilisaient 'à peine' comme quasi-synonyme de 'un peu' pouvaient trouver qu'il y avait entre les deux phrases une différence d'orientation argumentative (c'est moi qui le formule comme ça), et des personnes qui utilisaient 'à peine' systématiquement de manière standard pouvaient réponde qu'il n'y avait aucune différence. Visiblement, le fait de se mettre sur un niveau plus abstrait que l'usage spontané perturbait les sujets au point de donner des réponses contradictoires.

Le même type de problème pouvait se poser pour les réponses qui étaient censées être intuitives (les deux premières séries de questions). Est-ce que le simple fait de prendre un peu de recul par rapport à son propre usage de la langue serait susceptible d'altérer la réponse ? En effet, la personne même qui s'était excusée une fois oralement en énonçant la phrase (1) Je suis désolée, je suis à peine en retard ne reconnaissait pas du tout cette phrase dans le questionnaire. Elle a répondu B) : incorrect, on ne pourrait 
jamais entendre ça. Le fait de devoir se prononcer à l'écrit alors qu'il s'agit d'un usage majoritairement oral est également une source de biais possible dans les jugements.

Dans son article de 1975, William Labov souligne l'effet de l'examinateur ou de la situation d'examen sur les questionnés. Le jugement qu'ils émettent de leur propre usage de la langue peut être en nette contradiction avec leur comportement langagier spontané :

«Ce n'est pas un fait nouveau de dire que les gens affirment une chose et en font une autre. Dans nos recherches sociolinguistiques, nous n'avons pas été surpris de découvrir que les personnes qui affirment ne pas dire " ain't”, ni laisser tomber les $g$, ni prononcer de manière plus aiguë les /a/ brefs, les utilisent en fait dans la demiheure qui suit en discours non-surveillé. » (Labov, 1975)

Ou encore,

\begin{abstract}
« Nous avons donc été relativement surpris de trouver un grand nombre de cas où les sujets utilisaient des structures syntaxiques dans les formes les plus naturelles de l'interaction, après que leur introspection la plus consciencieuse avait montré que l'utilisation de ces formes était impossible pour eux. » (Labov, 1975)
\end{abstract}

Pour ces raisons, on doit être prudent face aux résultats d'un questionnaire comme celui que j'ai réalisé sur l'usage dialectal de l'opérateur 'à peine'. Il ne saurait refléter fidèlement et avec précision l'étendue de l'emploi que l'étude cible. Néanmoins, même si l'on est obligé de prendre les chiffres avec précaution, les tendances sont suffisamment claires, et, comme j'ai précisé plus haut, les résultats obtenus restent compatibles avec les hypothèses (H1) et (H2).

\title{
4 La description sémantique de la variante régionale de 'à peine'
}

J'ai montré dans les chapitres précédents que :

1. il était au moins rationnel de considérer qu'il existe un usage non-standard de l'expression 'à peine' en Franche-Comté, et que

2. il ne s'agissait pas d'un sens seulement un peu plus général ou plus spécifique par rapport au sens standard, comme c'est souvent le cas des emplois dialectaux, mais d'une unité linguistique qui a la même forme mais une signification fondamentalement différente.

En effet, pour reprendre la terminologie ducrotienne de la période des échelles argumentatives (Ducrot, 1980), le 'à peine' standard et le 'à peine' dialectal n'appartiennent pas à la même classe argumentative, c'est-à-dire, si les énoncés argumentatifs utilisant le 'à peine' standard s'orientent vers une conclusion $r$, les énoncés contenant le 'à peine' dialectal s'orientent vers une conclusion non-r. Il est difficilement concevable d'élaborer une seule description sémantique de 'à peine' qui puisse rendre compte à la fois des deux usages qui ont des comportements argumentatifs opposés.

De ce fait, on est contraint de considérer que le morphème 'à peine' tel qu'il est utilisé en Franche-Comté est un morphème différent, et ce malgré la filiation étymologique et la forme communes, et malgré le fait que les locuteurs francs-comtois n'en soient guère conscients. Un morphème différent exige une description différente de celle du morphème standard.

S'il est vrai que l'usage régional et l'usage standard ont des orientations argumentatives opposées, il n'en découle pas qu'ils sont symétriques. Quand Ducrot sépare un sens de 'à peine' qui se rapproche de 'seulement' et un autre qui ressemble à 'peu', cette distinction peut paraitre peu pertinente une fois que la description qu'il propose les réunit. Cette dernière est formulée alors en termes de présupposé et posé :

«Soit un énoncé $E$, décomposable en $X+Y+a ̀$ peine (avec à peine portant sur $Y$ ). [...] $E$ présuppose tout ce que poserait et présupposerait $X+Y$. Ce que pose $E$, maintenant, c'est que $Y$ est, parmi les termes de sa catégorie, le terme le plus fort que l'on puisse employer ici ; [...]» (Ducrot, $1972: 258)$.

La description en termes de contraintes sur les topoi que j'ai proposée dans 2.2 n'exige pas non plus que l'on sépare ces deux sens, du fait que, nous l'avons vu, 'à peine', 'peu' et 'seulement' imposent la même 
contrainte formelle sur la forme des topoi évoqués par le segment sur lequel ces opérateurs portent. Or, l'usage dialectal ne concerne que les cas où 'à peine' pourrait être remplacé par 'peu', la distinction reste donc ici pertinente.

Il y a deux manières d'appréhender la description de l'usage dialectal de 'à peine':

1. soit on considère que le 'à peine' franc-comtois a, tout comme l'opérateur standard, deux sens et une seule description doit les réunir,

2. soit on postule qu'en Franche-Comté, il existe deux opérateurs 'à peine': un standard qui est tronqué de son sens qui pourrait en faire un quasi-synonyme de 'peu', et un dialectal, qui est utilisé dans le sens de 'un peu'.

La première possibilité se heurte au même type d'obstacle que l'option de considérer l'opérateur standard et l'opérateur régional comme deux variantes du même morphème. Étant donné que l'usage franccomtois accepte à la fois des phrases comme (10) et des phrases comme (11) :

(11) Julie a à peine seize ans, elle est trop jeune pour se marier.

(12) Il fait à peine froid, je mets un pull plus chaud.

on est bien obligé d'admettre que l'emploi spécifiquement franc-comtois de 'à peine' exige une description à part.

\subsection{La description formelle}

Comme nous avons vu que la bizarrerie des énoncés utilisant un 'à peine' dialectal cesse si l'on remplace 'à peine' par 'un peu', il est rationnel d'envisager que la description du 'à peine' dialectal soit similaire à celle de 'un peu'. C'est ce que j'ai proposé plus haut en formulant l'hypothèse (H3).

Il reste donc à tester si la description de 'un peu' rend compte des mêmes contraintes topiques que 'à peine' en Franche-Comté.

La description de 'un peu' proposée par Raccah est la suivante (Raccah, 1990a) :

Dans les phrases comportant un 'un peu $X$ ', pour toute situation, le signe de l'antécédent du topos évoqué par X sera $+($ plus $)$.

Examinons la phrase

(13) Il y a à peine de vent, le linge va vite sécher.

Cette phrase a été jugée acceptable par la plupart des locuteurs francs-comtois interrogés.

Le topos évoqué est dans ce cas :

// Plus il y a du vent, plus vite le linge sèche//.

Il serait impossible de trouver un topos dont l'antécédent serait dans le champ '-‘. Si on prend un autre exemple qui a été accepté par la plupart des sujets francs-comtois,

(14) Le café est à peine trop chaud, il faut que tu attendes avant de le boire.

le topos mobilisé sera :

// Plus le café est chaud, moins on peut le boire//.

On peut constater que, tout comme 'un peu', le 'à peine' régional conditionne l'antécédent du topos (toujours en + ) mais pas le conséquent (peut être en + ou en -). La description peut donc être adaptée à l'opérateur 'à peine' franc-comtois : 
Dans le parler franc-comtois, il existe un morphème 'à peine' non standard. Dans les phrases comportant ce 'à peine' portant sur X, pour toute situation, le signe de l'antécédent du topos évoqué par X sera $+(p l u s)$

\subsection{Faut-il inclure la notion de degré dans la description?}

Une partie des sujets francs-comtois interrogés trouvait qu'entre Je suis à peine enrhumé et Je suis un peu enrhumé, il y avait une différence de degré. Il est difficile de décider si les personnes pensaient à un 'à peine' standard, ou un 'à peine' régional. Dans le premier cas, la différence d'orientation argumentative peut être prise par des locuteurs non spécialisés pour une différence de degré : en effet, être "à peine enrhumé » permet de conclure qu'on peut aller travailler, alors que « un peu enrhumé » ne peut être un argument que pour une conclusion du type « on doit rester à la maison ». La différence concernant les conséquences possibles peut faire croire que c'est la gravité de la maladie qui n'est pas du même degré. Pour traiter cette possiblité, l'AdL et la Sémantique des Points de Vue considèreraient que cette perception de différence n'est pas due à l'état du monde mais aux instructions que les éléments linguistiques donnent sur l'interprétation: ‘à peine' minimise les conséquences tandis que 'un peu' les renforce.

Dans le deuxième cas, le locuteur peut effectivement faire une différence de degré entre l'usage d'un 'à peine' régional et celui d'un 'un peu'. Les enjeux de l'étude ont été révélés à certains des sujets, qui ont pu donner librement leur avis après avoir rempli le questionnaire. Ceux qui ont admis l'existence d'un usage différent du standard, soutenaient que 'à peine' exprimait un degré plus faible que 'un peu'. En appliquant à ces observations le modèle des échelles argumentatives, on pourrait dire que pour eux, les deux opérateurs seraient sur la même échelle (appartiennent à la même classe argumentative), mais leur force argumentative serait différente. La méthode ducrotienne pour évaluer la force argumentative des mots consiste à les soumettre au "test de 'même'» ('même' ayant la propriété de mettre en évidence les rapports de force entre deux arguments de la même classe argumentative). Si l'intuition des locuteurs affirmant que 'à peine' exprime une quantité inférieure à celle de 'un peu' concerne bien leur forces argumentatives respectives, la phrase

(15) Il y a à peine de vent, même un peu.

doit être considérée comme acceptable pour les locuteurs francs-comtois.

L'acceptabilité de ce type de construction a été testée oralement et de manière informelle auprès des locuteurs francs-comtois; il semblerait que non seulement ces derniers ne l'utilisent pas, mais ils n'y trouvent pas d'interprétation possible. Ce qui confirmerait que la différence de degré perçue par ces mêmes locuteurs vient des situations d'énonciation dont une description pragmatique pourrait rendre compte.

\subsection{Vers une description plus complète}

Si l'on accepte, comme suggéré dans 1.1, qu'une description sémantique doit mettre en évidence des instructions linguistiques constantes à la construction du sens, la description formelle proposée plus haut satisfait cette exigence. Ce type d'analyse en termes de contraintes topiques a néanmoins des limites: étant donné le nombre fini des formes des topoi, les contraintes possibles que les différents articulateurs peuvent imposer sont également de nombre limité : elles portent soit sur l'antécédent, soit le conséquent du topos, soit sur les deux à la fois, les signes des champs topiques peuvent être soit + , soit -, ce qui laisse 8 combinaisons possibles. Le modèle topique comporte d'autres éléments qui peuvent également intervenir en tant que contraintes stables descriptibles, comme par exemple « $\mathrm{r}$ » (orientation argumentative, ce qui est une valeur des champs topiques) qui apparaît dans la description de l'opérateur 'trop' (Raccah, 2005), mais tous les articulateurs ne comportent pas nécessairement une telle spécification. Ce qui revient à présumer que tous les opérateurs et autre connecteurs peuvent être classés dans 8 grandes « familles ». Cette hypothèse n'est pas en soi absurde - en effet, rien n'empêche que le 
modèle prévoie une telle classification - mais, dans la plupart des cas des articulateurs déjà décrits en ces termes, la description ne va pas plus loin que de déterminer l'appartenance à une de ces classes.

Or, si l'on suppose que la différence entre deux articulateurs appartenant à la même catégorie est de nature sémantique et non pas pragmatique, il serait souhaitable que la description puisse rendre compte de cette différence. Lorsque le cadre du modèle topique ne permet pas de spécification différentielle, on peut envisager de croiser la description avec d'autres, issues d'autres modèles sémantiques instructionnels, comme par exemple avec le modèle polyphonique, comme le proposent (Anscombre \& Ducrot, 1983: 174-179) ou (Raccah, 2006).

L'objectif de cet article était avant tout de confirmer de manière formelle un fait linguistique décelé par l'intuition, en se servant d'un cadre conceptuel susceptible de rendre compte dudit fait. Ce qui devait être mis en évidence, c'est le problème d'interprétation de certaines occurrences dialectales de l'expression 'à peine' témoigné par des locuteurs de français standard. Il a été montré que la différence sémantique entre la version standard et la version dialectale résidait dans leurs orientations argumentatives respectives, le cadre topique était donc un outil efficace pour analyse du phénomène. Si l'opérateur dialectal - tout comme son homonyme en français standard - peut sans doute être décrit de manière plus exhaustive en combinant plusieurs modèles instructionnels, cela dépasse l'ambition de ce travail dont le but a été atteint.

\section{Perspectives sociolinguistiques}

La présente analyse n'a comme objectif que de décrire une particularité sémantique de l'usage régional de l'expression 'à peine'. De là à ce qu'elle puisse s'inscrire dans les études sociolinguistiques proprement dites sur le parler franc-comtois, comme je l'ai précisé plus haut, elle devrait être complétée par un volet contenant des analyses plus précises sur les zones géographiques et les types de locuteurs concernés par cet usage. Comme je l'avais mentionné dans 3.3, le petit échantillon de réponses semble confirmer l'hypothèse selon laquelle c'est la population à la fois rurale et peu lettrée qui produit le plus couramment l'emploi dialectal de 'à peine', ce qui est parfaitement prévisible dans tout sondage portant sur les emplois régionaux. Cependant, le nombre très limité des questionnés non Franc-Comtois vivant en Franche-Comté, et des Franc-Comtois vivant ailleurs ne m'a permis de dégager aucune tendance significative de leur manière d'intégrer, ou, au contraire, d'abandonner l'usage régional. Les résultats sont donc appelés à être affinés du point de vue sociolinguistique.

Par ailleurs, s'agissant d'un usage essentiellement oral, des tests oraux pourraient être effectués auprès des locuteurs avec des résultats plus exacts. On pourrait notamment tenter de déterminer si des marques prosodiques permettent de distinguer l'emploi dialectal de l'emploi standard chez les 'bilingues'.

Il serait également intéressant de voir si cet usage a quelque rapport avec les patois locaux. L'interrogation des patoisants pourrait mettre en évidence la présence ou non d'une expression correspondant à 'à peine' régional, distincte de l'expression correspondant de 'à peine' standard (si elle existe). Il s'agit donc d'un domaine où la coopération entre sémantique et sociolinguistique pourrait s'avérer particulièrement fructueuse. 


\section{Annexe : Le questionnaire sur l'usage de ‘à peine’ en Franche-Comté}

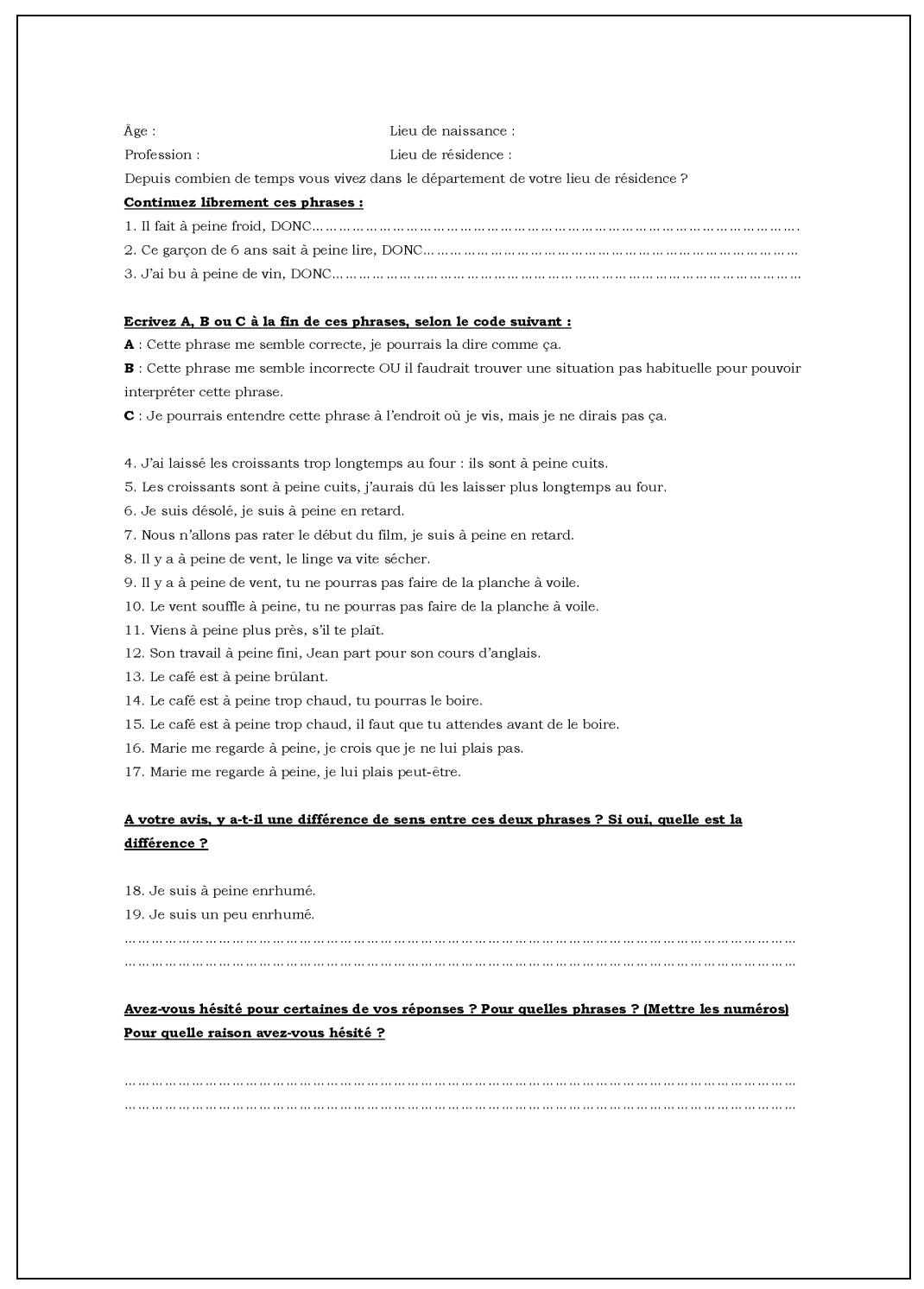

\section{Références bibliographiques}

Anscombre, J-C., Ducrot, O. (1983). L'argumentation dans la langue. Liège : Pierre Mardaga.

Anscombre, J-C. (dir.) (1995). Théorie des topoi. Paris : Kimé.

Bruxelles, S., Ducrot, O., Raccah, P-Y. (1994). Argumentation et champs topiques lexicaux. Cahiers de Praxématique 21, 88-104.

Ducrot, O. (1972). Dire et ne pas dire. Paris : Hermann.

Ducrot, O. (1980a). Les échelles argumentatives. Paris : Minuit.

Ducrot, O. et alii. (1980b). Les mots du discours. Paris : Minuit.

Ducrot, O. (1983). Opérateurs argumentatifs et visée argumentative. Cahiers de linguistique française 5, 7-36.

Ducrot, O. (1988). Topoi et formes topiques. Tokyo : Bulletin d'études de linguistique française 22, 1-14. 
Fauconnier, G. (1984). Espaces mentaux. Paris : Les Éditions de Minuit.

Labov, W. (2001). Qu'est-ce qu'un fait linguistique ? Marges linguistiques 1, 25-68. Original : Labov, W. (1975). What is a linguistic fact? Lisse - The Peter de Ridders Press.

Némo, F., Berruecos, L. (2003). Indexicalité, unification contextuelle et constitution extrinsèque du référent. Langages 150, 88-105.

Raccah, P-Y. (1990a). Modelling argumentation and modelling with argumentation. Bruxelles : Argumentation 4, $447-483$

Raccah, P-Y. (1990b). Signification, sens et connaissance : une approche topique. Cahiers de linguistique française $11,179-198$

Raccah, P-Y. (1998). L'Argumentation sans la preuve : prendre son biais dans la langue. Interaction et cognition II$1 \& 2,237-264$

Raccah, P-Y. (2002). Lexique et idéologie : les points de vue qui s'expriment avant qu'on ait parlé. In Carel M. (éd.), Les Facettes du dire, Hommages à Oswald Ducrot, Paris : Kimé. 241-268.

Raccah, P-Y. (2005). Une description de l'excessivité en sémantique des points de vue. Travaux de linguistique du CERLICO 18, 171-190.

Raccah, P-Y. (2006). Polyphonie et argumentation: des discours à la langue (et retour...). In Simonffy, Zs. (éd.) : L'un et le multiple. Budapest : TINTA Könyvkiadó, 120-152

Raccah, P-Y. (2011). La sémantique des points de vue, un outil utile ? Conférence à l'Université d'Orléans, 24 janvier 2011.

\footnotetext{
${ }^{1} \mathrm{Du}$ fait que je n'ai pas cherché à délimiter de manière précise l'étendue géographique de cet usage, je ne peux pas affirmer qu'il n'existe pas ailleurs, par exemple dans les départements limitrophes. Il semblerait qu'il n'est pas attesté dans les Vosges, mais je n'ai pas de données concernant la Côte d'Or ou l'Ain. En revanche, j'ai recueilli des données positives dans les quatre départements de Franche-Comté ; dans le présent article, je me réfèrerai donc à cet usage comme usage franc-comtois, sans pour autant exclure la possibilité qu'il puisse être attesté ailleurs.
}

${ }^{2}$ Pour (1) : Pontarlier, Doubs. Pour (2) : Besançon, Doubs. Pour (3) : Landresse, Doubs.

${ }^{3}$ Dans la linguistique cognitive américaine, les unités de langue renvoient à des états mentaux.

${ }^{4}$ Il précise qu'il ne s'agit pas nécessairement du segment qui suit le morphème 'à peine' : il peut y avoir des énoncés de la phrase «Le dimanche, Jean voit à peine ses enfants » où 'à peine' porte sur 'voir' et d'autres où il porte sur 'ses enfants'. Il appelle segment incident le segment sur lequel 'à peine' porte. Pour permettre des descriptions plus formelles, j'utiliserai dans cet article la forme 'à peine $X$ ' où $\mathrm{X}$ reste toujours le segment incident.

${ }^{5}$ Consulté sur Internet le 12 mars 2012. http://linguistes.fr/public/Raccah/Liste.html

${ }^{6}$ Voir aussi (Raccah, 1998), pour une démonstration plus formelle.

${ }^{7}$ L'AdL utilise des astérisques pour marquer les enchaînements qui ne paraissent pas possibles - ou qui paraissent bizarres - dans le discours. Néanmoins, on ne peut pas dire qu'ils soient « sémantiquement incorrects » de la même façon que la grammaire générative marquait les phrases agrammaticales. On peut presque toujours trouver des situations où la phrase en question devient énonçable : l'astérisque indique que ce qui pourrait rendre énonçable cette phrase vient de la situation (Ducrot, $1983: 10$ ).

${ }^{8}$ Pour des présentations plus amples des tenants et aboutissants de ce modèle, voir (Ducrot, 1988), (Anscombre, 1995), (Bruxelles, Ducrot \& Raccah, 1994), (Raccah, 1998).

${ }^{9}$ En raison du 'mais' qui les relie, l'interlocuteur comprendra l'énoncé entier comme un refus à la proposition de promenade (Anscombre \& Ducrot, 1983).

${ }^{10}$ Je reprends ici, en l'adaptant à 'peu' et 'un peu', la description que (Raccah, 1990) fournit de 'little' et 'a little'. Raccah n'a pas publié de description de 'peu' et 'un peu' mais a souvent précisé oralement lors de ses séminaires, qu'il se trouve que cette description est également applicable aux opérateurs français 'peu' et 'un peu'. La description 
originale est la suivante : "little requires a topos whose first half (call it its antecedent) is a 'minus-field', while $a$ little requires a topos whose antecedent is a 'plus-field' » (Raccah, 1990). 